\title{
A new approach to follow a single extracellular vesicle-cell interaction using optical tweezers
}

llaria Prada1 , Ladan Amin², Roberto Furlan³, Giuseppe Legname ${ }^{2}$, Claudia Verderio ${ }^{1,4}$, and Dan Cojoc ${ }^{5}$ ${ }^{1}$ CNR Institute of Neuroscience, Milan, Italy, ${ }^{2}$ Department of Neuroscience, Scuola Internazionale Superiore di Studi Avanzati (SISSA), Trieste, Italy, ${ }^{3}$ Institute of Experimental Neurology, Division of Neuroscience, San Raffaele Scientific Institute, Milano, Italy, ${ }^{4}$ IRCCS Humanitas, Rozzano, Italy, and ${ }^{5}$ CNR - Institute of Materials, Trieste, Italy

BioTechniques 60:35-41 (January 2016) doi 10.2144/000114371

Keywords: optical tweezers; microvesicles (MVs); microglia; astrocytes; extracellular vesicles (EVs)

Supplementary material for this article is available at www.BioTechniques.com/article/114371.

Extracellular vesicles (EVs) are spherical membrane structures released by most cells. These highly conserved mediators of intercellular communication carry proteins, lipids, and nucleic acids, and transfer these cellular components between cells by different mechanisms, such as endocytosis, macropinocytosis, or fusion. However, the temporal and spatial dynamics of vesicle-cell interactions still remain largely unexplored. Here we used optical tweezers to drive single EVs produced by microglial cells onto the surface of astrocytes or microglia in primary culture. By visualizing single EV-cell contacts, we observed that microglial vesicles displayed different motilities on the surface of astrocytes compared with microglia. After contact, EVs positioned on astrocytes displayed some minor oscillatory motion around the point of adhesion, while vesicles dragged to microglia displayed quite regular directional movement on the plasma membrane. Both the adhesion and motion of vesicles on glial cells were strongly reduced by cloaking phosphatidylserine (PS) residues, which are externalized on the vesicle membrane and act as determinants for vesicle recognition by target cells. These data identify optical manipulation as a powerful tool to monitor in vitro vesicle-cell dynamics with high temporal and spatial resolution and to determine in a quantitative manner the contribution of surface receptors/extracellular protein ligands to the contact.

Cells release into the extracellular environment diverse types of extracellular vesicles (EVs), which bud from the plasma membrane [ectosomes or microvesicles (MVs)] or result from exocytosis of multivesicular bodies (exosomes) (1). EVs carry a broad repertoire of donor-cell components, including proteins, lipids, (micro)RNAs, and DNAs, and deliver their cargo to recipient cells, thereby trafficking complex intercellular signals (2). To exchange molecular information, EVs enter cells by various endocytic mechanisms (3), such as clathrin- or caveolin-dependent endocytosis, macropinocytosis (4), or phagocytosis (5), followed by fusion with the endosomal membrane. Alternatively, EVs deliver their cargo through direct fusion with the plasma membranes of recipient cells $(6,7$, and reviewed in 8). However, delivery of cargo is not always required to elicit a cellular response. Interaction of EV surface proteins/ligands with molecules exposed on the plasma membranes of target cells may indeed be sufficient to activate signaling events and alter the function of recipient cells $(9,10)$.

Under pathological conditions, production of EVs increases, and EVs become vehicles of pathogenic cargo [e.g., aggregating proteins in degenerative diseases, oncoproteins in cancer (11-17), or inflammatory cytokines in neuroinflammatory diseases (18-21)]. Because of their small size (<100 nm for exosomes, $<1000 \mathrm{~nm}$ for MVs) disease-promoting EVs can move from the site of discharge and mediate communication with distant cells (8).

Our previous work indicates that MVs released from the surface of reactive microglia, the immune cells of the brain (22), induce an inflammatory reaction in recipient glial cells, both microglia and astrocytes (23), the most numerous and diverse glial cells in the nervous system (24). However, microglia-derived MVs and exosomes may interact and signal to astrocytes or microglia by distinct mechanisms. In microglia, the inflammatory reaction evoked by MVs is associated with MV uptake into cells and the transfer of mRNA for the inflammatory cytokine IL-1 $\beta$ (23). Conversely, little or no uptake of MVs or exosomes occurs in astrocytes, and no evidence for microglia-to-

Here we present a new approach for positioning individual extracellular vesicles onto the membranes of cells using optical tweezers and monitoring the vesicle-cell dynamics in vitro. Our technique allows quantitative determination of the contribution of surface receptors and extracellular protein ligands to the contact. 
astrocyte transfer of nucleic acids through EVs has been presented $(4,18,19)$.

Optical tweezing allows trapping and precise manipulation of small particles the size of EVs and is emerging as a promising technique to deliver molecules to cells in a highly controlled manner $(25,26)$. Our previous work indicates that beads carrying active brain-derived neurotrophic factor (BDNF) or liposomes loaded with guidance molecules can be optically manipulated to deliver stimuli to neurons with great spatial and temporal resolution $(27,28)$. To better define how microglia-derived MVs, the vesicle population more extensively characterized in our previous studies, interact with glial cells, we exploited optical manipulation and live-cell microscopy to directly drive MVs to astrocytes or microglia in primary culture. We show that microglia-derived MVs displayed distinct behaviors when delivered to the two glial cell types. MVs promptly adhered to both glial cell types; however, after adhesion (i.e., binding of the MVs to cells), MVs remained stuck to the plasma membranes of astrocytes, whereas they moved slowly along the surface of microglia. In addition, we show that cloaking phosphatidylserine (PS) residues on $\mathrm{MV}$ s with annexin $\mathrm{V}$ inhibited MV-cell contact and reduced MV movement on the microglial surface. These findings demonstrate optical manipulation to be an ideal method to directly image EV-cell interactions and to determine in a quantitative manner the contribution of surface co-receptors and extracellular protein modulators to the contacts.

\section{Materials and methods}

Animals

All of the experimental procedures followed the guidelines established by European legislation (Directive 2010/63/EU) and Italian Legislation (L.D. no 26/2014). All efforts were made to minimize the number of animals used and their suffering.

Primary glial culture

Mixed glial cell cultures, containing both astrocytes and microglial cells, were established from rat Sprague-Dawley pups (P2) (Charles River, Lecco, Italy). Briefly, after dissection, hippocampi and cortices were dissociated by treatment with trypsin (0.25\%) and DNase-I (Sigma-Aldrich, St. Louis, MO) for $15 \mathrm{~min}$ at $37^{\circ} \mathrm{C}$, followed by fragmentation with a firepolished Pasteur pipette. Dissociated cells were plated on poly-L-lysine-coated T75 flasks in minimal essential medium (E-MEM, Invitrogen, Life Technologies, Carlsbad, CA) supplemented with 20\% fetal bovine serum (FBS) (Gibco, Life Technologies, Carlsbad, CA) and glucose (5.5 g/L). To obtain a pure astrocyte monolayer, microglial cells were harvested from 10-14-day-old cultures by orbital shaking for $30 \mathrm{~min}$ at $1300 \mathrm{rpm}$. Astrocytes were trypsinized and re-plated onto poly-L-lysine-coated glass coverslips, while shaken microglia were re-plated on polyL-ornithine-coated tissue culture dishes or glass coverslips.

Microvesicle isolation and treatment Microglia were exposed to $1 \mathrm{mM}$ ATP (SigmaAldrich) for 30 min in Krebs-Ringer's HEPES solution $(\mathrm{KRH})(125 \mathrm{mM} \mathrm{NaCl}, 5 \mathrm{mM} \mathrm{KCl}, 1.2$ $\mathrm{mM} \mathrm{MgSO}_{4}, 1.2 \mathrm{mM} \mathrm{KH}_{2} \mathrm{PO}, 2 \mathrm{mM} \mathrm{CaCl}_{2}, 6$ mM D-glucose, 25 mM HEPES/NaOH, pH 7.4). Conditioned $\mathrm{KRH}$ was collected and pre-cleared from cells and debris by centrifugation at $800 \times g$ for 10 min (twice). Very large MVs were then pelleted from the supernatant by centrifugation at $10,000 \times g$ for $30 \mathrm{~min}$ and used immediately after isolation.
In a set of experiments, isolated MVs were incubated for $30 \mathrm{~min}$ at room temperature with $0.84 \mu \mathrm{g} / \mathrm{mL}$ annexin $\vee$ (Sigma-Aldrich) to cloak PS residues before being delivered by optical tweezers.

\section{Optical tweezers}

Immediately before recording, recipient glial cells $\left(1 \times 10^{5}\right)$ plated on glass coverslips were washed with $\mathrm{KRH}$ and kept in $100 \mu \mathrm{L}$ of this solution in the temperaturecontrolled recording chamber of an inverted microscope (Nikon Eclipse TE-2000-E; Nikon Instruments Spa, Florence, Italy). In both microglia and astrocytes, constitutive shedding does not occur at a high rate, and it is extremely difficult to detect locally born MVs floating in the medium after washing of the cultures. MVs produced by $3 \times 10^{5}$ microglia were then added to glial cells, and as soon as MVs appeared in the recording field, they were captured by optical tweezers to be driven onto cells. Only one field per coverslip was analyzed in order to minimize the risk of capturing locally born MVs, which may constitutively accumulate in the medium over time. An infrared (IR) laser beam (1064 nm, CW) was collimated and coupled into the optical path of the microscope. The trapping beam was directed into the microscope lens (Nikon 60x, NA 1.25) by a dichroic mounted above the fluorescence cube (29). A single MV was trapped and positioned on a selected cell by moving the cell stage horizontally and the microscope lens axially, as shown in Figure 1. After $~ 30$ $s$ of contact, the laser was switched off to prove MV-cell interaction. During the experiments, the cells were monitored by timelapse phase contrast imaging using a digital camera (Orca Flash 4.0; Hamamatsu, Japan) at a frame rate of $2 \mathrm{~Hz}$.

Table 1. Adhesion and movement of microglial microvesicles (MVs) on microglia or astrocytes.

\begin{tabular}{|c|c|c|}
\hline & $\begin{array}{l}\text { Recipient } \\
\text { MICROGLIA }\end{array}$ & $\begin{array}{c}\text { Recipient } \\
\text { ASTROCYTES }\end{array}$ \\
\hline \multicolumn{3}{|l|}{ Adhesion } \\
\hline Control MVs & $11 / 14$ & $7 / 11$ \\
\hline Annexin V-coated MVs & $4 / 10$ & $2 / 12$ \\
\hline \multicolumn{3}{|l|}{ Movement } \\
\hline Control MVs & $8 / 11$ & $1 / 7$ \\
\hline Annexin V-coated MVs & $1 / 5$ & $0 / 2$ \\
\hline \multicolumn{3}{|c|}{$\begin{array}{l}\text { Values indicate the number of MVs adherent to } \\
\text { glial cells out of the total number of MVs driven } \\
\text { to microglia or astrocytes by optical manipu- } \\
\text { lation in control conditions or after treatment } \\
\text { with annexin } V \text { (adhesion). Values indicate the } \\
\text { number of MVs moving on the surface of glial } \\
\text { cells out of the total number of MVs adherent to } \\
\text { microglia or astrocytes under control conditions } \\
\text { or after treatment with annexin } V \text { (movement). }\end{array}$} \\
\hline
\end{tabular}

Figure 1. Schematic representation of microvesicle (MV) delivery to glial cells by optical manipulation (tweezers). MV is first trapped above the cells by the IR laser tweezers (A). The stage is then moved in the horizontal plane (X-Y axes) and the objective/trap is moved axially (Z axis) to set the MV in contact with the cell (B). After $30 \mathrm{~s}$, the trapping laser is switched off (C) to check whether MV adheres to the cell membrane.
B
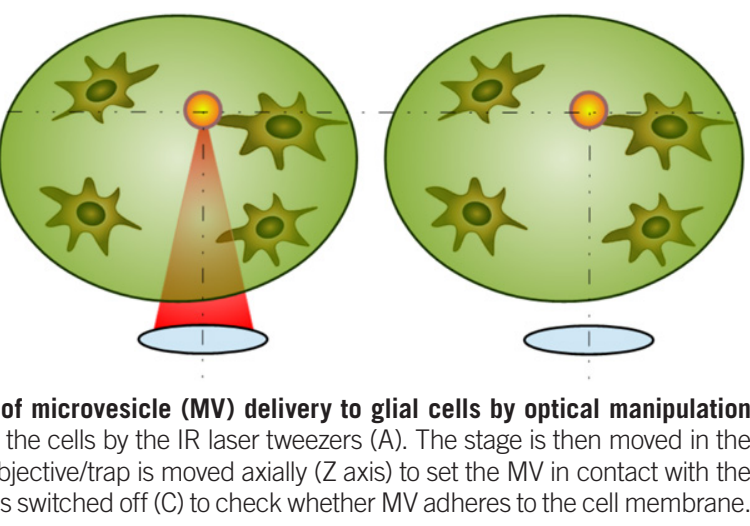

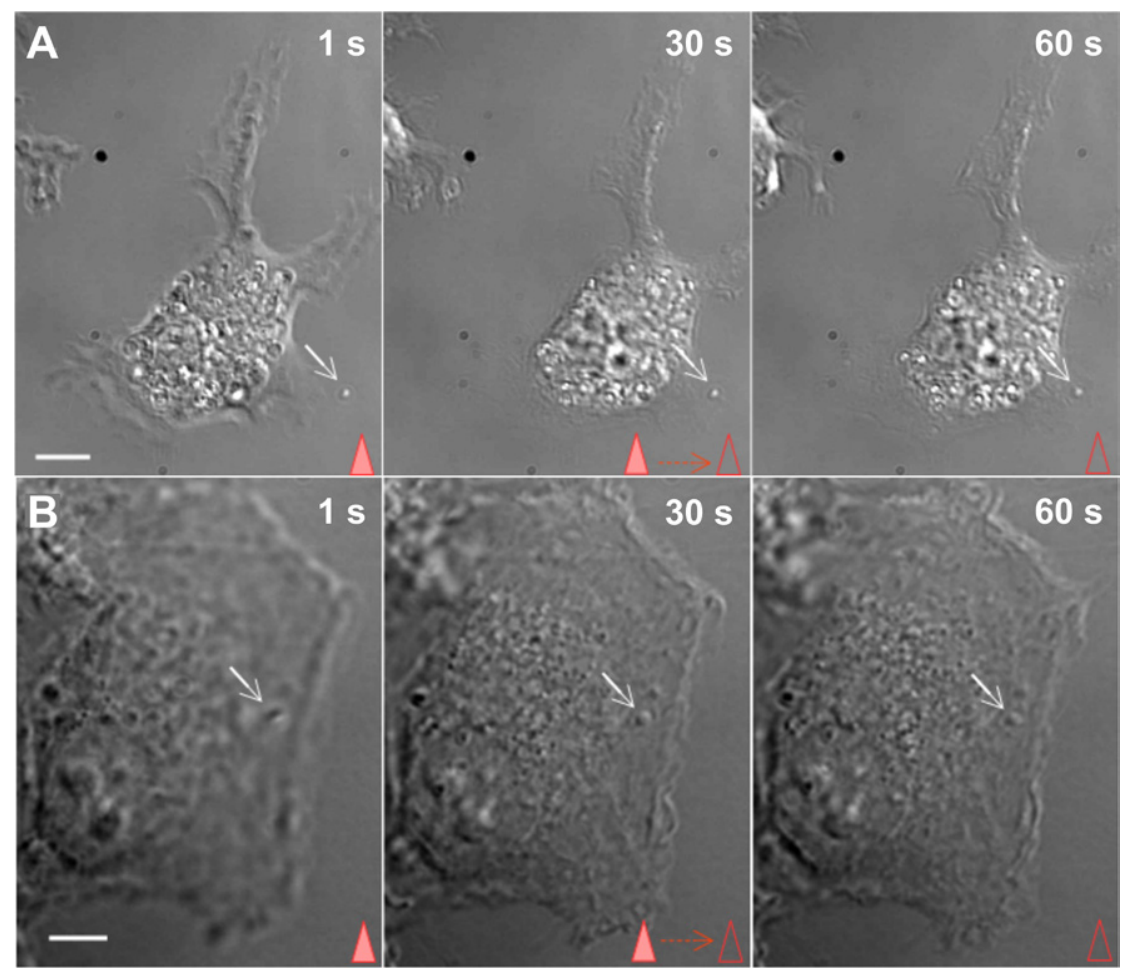

Figure 2. Imaging of single microvesicle (MV) adhesion to recipient microglial cells or astrocytes.

(A) The microglial MV was trapped above a microglia cell (not in focus) at $t=1 \mathrm{~s}$ and then positioned on the cell membrane by moving up the cell. The laser trap was switched off at $t=30 \mathrm{~s}$. Note that the $M V$ remained stuck in the same position $(t=60 \mathrm{~s}$ ), indicating $M V$ adhesion to the plasma membrane. White arrows in the phase contrast images point to the MV, while triangles indicate the trapping laser status: filled-ON, empty-OFF. (B) Sequence of phase contrast images showing a representative microglial MV driven to an astrocytic cell following the procedure described in (A). Scale bar: $5 \mu \mathrm{m}$.
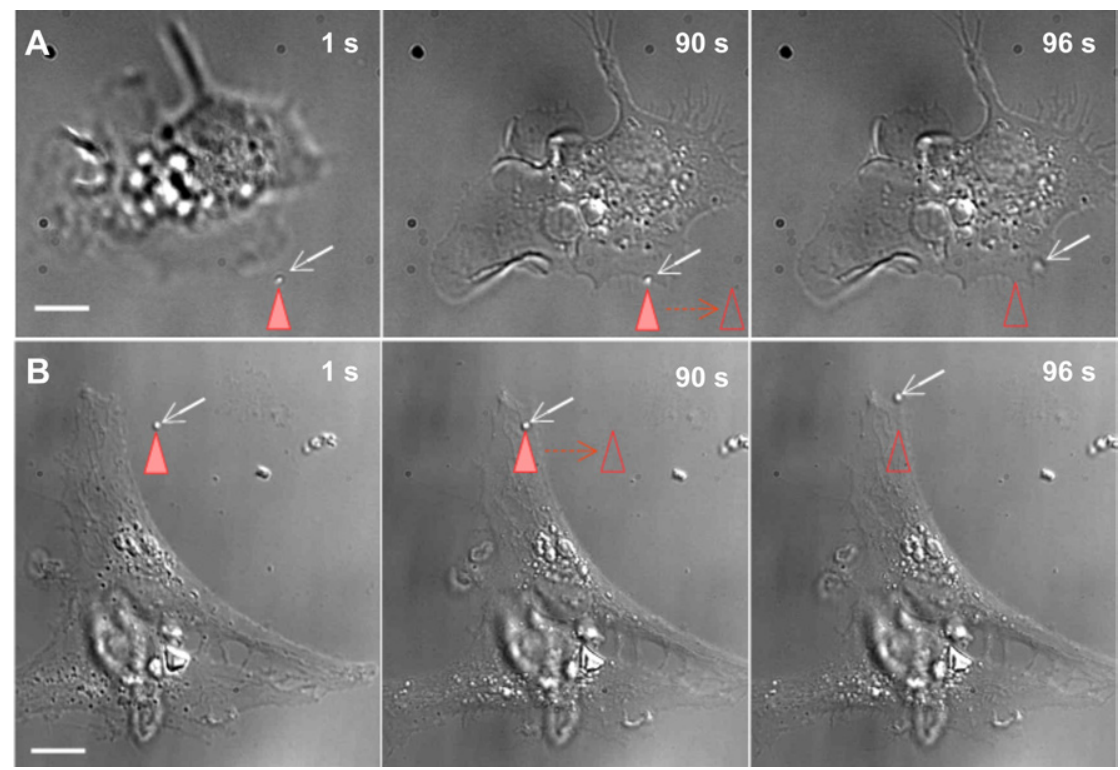

Figure 3. Cloaking phosphatidylserine (PS) residues on microvesicle (MV) membrane decreases MV adhesion to glial cells. (A) Microglial MVs were incubated with the PS ligand annexin V for 30 $\mathrm{min}$ and then added to the culture medium of glial cells. Images in (A) show the typical behavior of annexin V-treated MVs driven to microglia by optical tweezers. The MV was trapped above a microglia cell at $t=1 \mathrm{~s}$, and then positioned on the cell membrane by moving up the cell. The MV was kept in contact with the cell for $60 \mathrm{~s}$, and then the laser trap was switched off $(t=90 \mathrm{~s})$. The image taken at $t=96 \mathrm{~s}$ (out of focus) shows that the MV moved away from the trap position. (B) Representative sequence of images of a microglial MV, treated with annexin $\mathrm{V}$, positioned on an astrocyte, following the procedure described in $(A)$. White arrows in $(A)$ and $(B)$ point to the $\mathrm{MV}$, while triangles indicate the trapping laser status: filled-ON, empty-OFF. Scale bar: $5 \mu \mathrm{m}$.
Microvesicle position tracking

MV position was determined for each video frame [2 frames per second (fps)] using a custom MATLAB code (it.mathworks.com). To characterize the MV displacement on the cell, 2 distances were then calculated: the distance of the MV from the initial position and the length of the path traveled by the MV in 300 s. Moving MVs showed a net displacement from the point of adhesion of $>2 \mu \mathrm{m}$.

\section{Results and discussion}

Microglia-derived microvesicles efficiently adhere to glial cells MVs produced by primary rat microglia upon ATP stimulation were isolated from the cell supernatant by ultracentrifugation at $10,000 \times g$ after pre-clearing from cells and debris (modified from Reference 30 ). Exposure to ATP favors shedding of MVs from the microglial surface versus exosome release from the endocytic compartment (30). After isolation, MVs were added to the culture medium of astrocytes or microglia in the recording chamber of an inverted microscope, equipped with IR laser tweezers. MVs diffusing in the medium were captured by the optical tweezers and positioned onto cells as illustrated in Figure 1. Briefly, single MVs, still suspended in the extracellular medium, were trapped by the IR laser tweezers above target glial cells in the microscope field (Figure 1A). The stage was then moved in the horizontal plane (XY/black arrow), and the objective/ trap was moved axially (Z) to place the MVs in contact with the cells (Figure 1B). After 30 $\mathrm{s}$, the trapping laser was switched off (Figure $1 C)$, and the interactions between single MVs and cells were monitored by collecting phase contrast images (2 fps). Using this approach, we found that $79 \%$ and $64 \%$ of microgliaderived MVs adhered to microglia and astrocytes, respectively, remaining stuck to the place where they were positioned for the following $30 \mathrm{~s}$ (Table 1). Examples of MV adhesion to microglia (Figure 2A) or astrocytes (Figure 2B) are shown in Figure 2 and the associated Supplementary Movies S1 and S2. MV-cell interactions were quite strong, since MVs showed resistance to recapturing by the IR laser tweezers, as assessed by moving the laser trap up and down.

\section{Externalized phosphatidylserine is} involved in microvesicle-cell contacts Previous evidence has indicated that PS externalized on the surface of EVs is a determinant for EV recognition on recipient cells through interaction with PS receptors and 

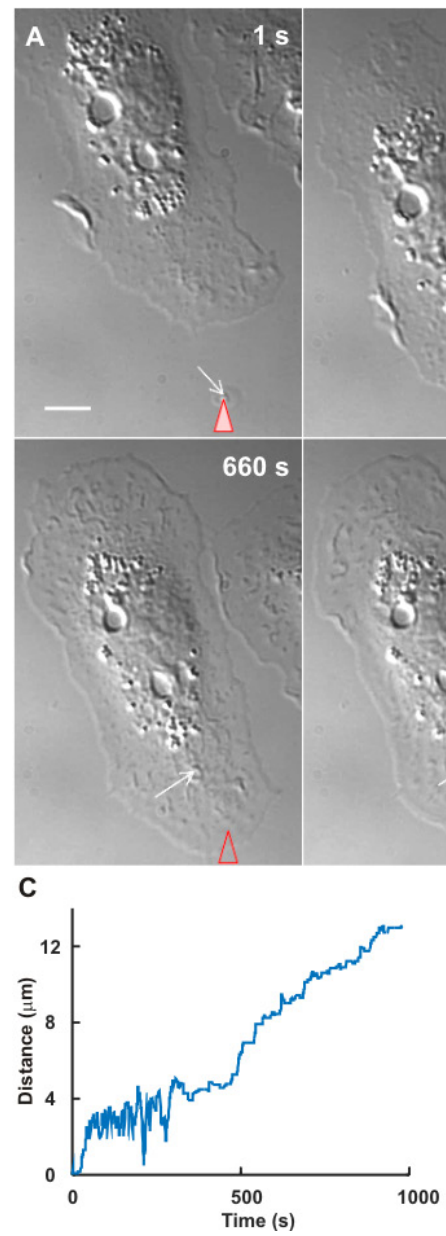
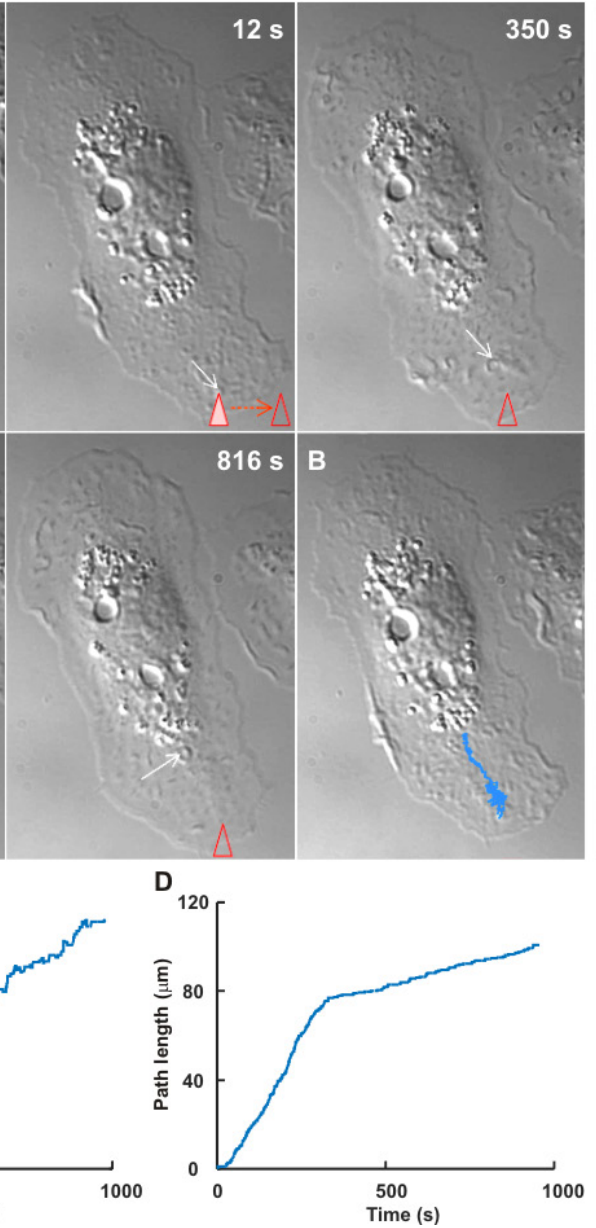
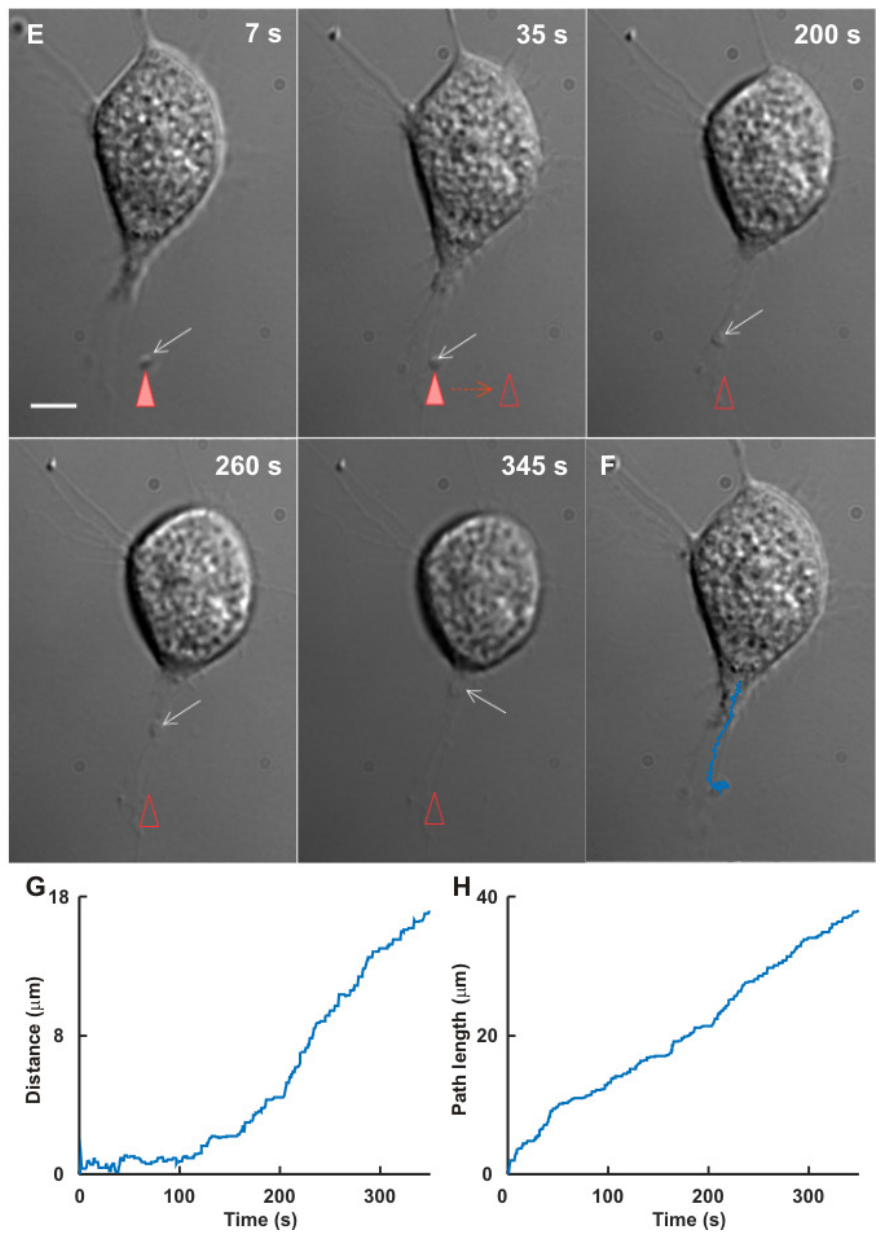

Figure 4. Interaction between single microglial microvesicles (MVs) and microglia: adhesion and transport. Two examples of microglia MVs trapped by the laser tweezers ( $\mathrm{t}=1 \mathrm{~s}$ ) and positioned on microglia. The MVs not only adhered to the cell surface but also moved toward the cell soma (A and E). Scale bar: 5 $\mu \mathrm{m}$. The trajectory of the MVs is represented in blue, superposed on the image of the cell at $t=30 \mathrm{~s}(\mathrm{~B}$ and $\mathrm{F})$. The distance traveled from the initial to the actual position of the MV during the experiment is plotted in (C) for $(A)$, in (G) for (E), and the path length of the MV in (D) for (A) and in (H) for (E).

that it controls the transfer of MV cargo to acceptor cells (31). In addition, we recently showed that cloaking PS residues on MVs with annexin $\mathrm{V}$, a high affinity ligand of PS, strongly decreased the modulation of excitatory transmission induced by microglial MVs (9).

To assess the contribution of PS to MV contact with glial cells, we treated MVs with annexin $\mathrm{V}$ before driving them onto glia by optical manipulation. Annexin V-treated MVs were kept in contact with acceptor glial cells for $60 \mathrm{~s}$ (i.e., twice the time of control MVs). However, when the laser was switched off, approximately two-thirds of the MVs moved away from the trap position in $10 \mathrm{~s}$, indicating that they did not adhere to target glial cells (Figure 3, A and B). Cloaking PS with annexin $V$ decreased the percentage of MVs that adhered to microglia and astrocytes to $40 \%$ and $17 \%$, respectively (Table 1 ). Repeated positioning of MVs onto acceptor glial cells confirmed impaired adhesion of annexin V-treated MVs (Figure 3B, Supple- mentary Movie S3). These data confirm an important role for externalized PS in MV-cell contact. However, they also suggest that PS is not essential for MV recognition, as a significant fraction of MVs still adhere to the glial surface after annexin V-treatment. Thus, other surface molecules likely contribute to the interaction between microglia-derived MVs and glial cells. Additional experiments are required to clarify whether MVcell contact is also associated with lipid exchange between the vesicular and cellular membranes.

Distinct motions of microglia-derived microvesicles on microglia and astrocytes

By monitoring the dynamics of MV-microglia interaction within periods of 20-30 min, we found that most MVs moved from the trap position after adhesion and displayed a net directional movement on the microglial surface toward the nuclear region (Figure 4, Supplementary Movies S4 and S5).
Movement occurred either along microglial processes or on flat lamellipodia. Although the resolution of phase contrast imaging did not allow discrimination between intra- and extra-MV trafficking, MVs appeared to surf on the plasma membrane of microglia, and their motion could be hampered by a new IR laser trapping. Tracking of single particles along the microglia surface showed that MV distances from the initial position increased with time. The distance reached by the MVs after the first 5 min of contact ranged from 2.2 to $17 \mu \mathrm{m}$ (mean $\pm \mathrm{SD}=6 \pm 5 \mu \mathrm{m}, n=7$ ). However, plots of the path length traveled by MVs as a function of time revealed that MV velocity was almost constant during the first 5 min of recording (2 examples are shown in Figure 4, $\mathrm{D}$ and $\mathrm{H}$ ), with the speed ranging from 50 to $280 \mathrm{~nm} / \mathrm{s}$ (mean $\pm \mathrm{SD}=117 \pm$ $75 \mathrm{~nm} / \mathrm{s}$ ). Interestingly, the velocity of MV movement on microglia is in the same range of that reported for adenoviral particles, which bind to cell surface receptors (32). It also resembles the speed of retrograde 
actin flow (33), which regulates the lateral diffusion of transmembrane proteins linked to actin (34). Given that both viruses and EVs are recognized on cells by surface receptors (35,36, and this study), the possibility arises that the motion of EVs outside microglia may be caused by the lateral diffusion of EV receptors, as previously suggested (37). Alternatively, movement of microglial MVs on the extracellular surface may be driven by a gradient of adhesion molecules to which MVs transiently bind, resembling the haptotactic motion of a negatively charged lipid vesicle on a positively charged lipid bilayer (38).

MV movement was often followed by disappearance of the MV (9 of 11), likely due to MV internalization into microglia. Consistent with this possibility, our previous evidence showed that microglial MVs can be taken up into microglia, becoming inaccessible to surface markers (18). Alternatively, MV disappearance could result from full fusion of MVs with the plasma membrane of microglia. Time-lapse confocal analysis of MVs labeled with self-quenching concentrations of membrane dyes, such as R18 dye (37) and/or retrospective staining of MVs after live-cell recordings will help to clarify this issue.

Markedly different dynamics were observed for the interaction of microglial MVs with astrocytes. Most MVs driven by $\mathrm{IR}$ tweezers onto astrocytes remained stably anchored to the astrocyte surface for tens of minutes, showing minor oscillatory motion around the point of adhesion. Only 1 of 7 observed MVs displayed a net directional movement along the astrocyte surface, at a low speed of $40 \mathrm{~nm} / \mathrm{s}$ (Table 1). In addition, microglial MVs never disappeared after contact with astrocytes. These findings rule out the possibility that MVs undergo full fusion with the plasma membrane or rapid internalization inside astrocytes (during
20-25 min of recording). Consistent with this, recent studies showed that exosomes released by glial cells are taken up through macropinocytosis by microglia but not by astrocytes (4).

Overall, the present study adds to our knowledge of the mechanisms of intercellular communication between glial cells mediated by MVs. Importantly, it validates optical tweezers as a powerful tool to explore the dynamics of MV trafficking outside cells and to quantify the contribution of surface molecules to MV-cell interactions.

Given that microglial MVs become the vehicles of pathogenic cargo proteins $(20,21)$ in neurodegenerative diseases, exploitation of optical manipulation will be crucial for a better understanding of how microglial MVs interact with neurons and oligodendrocytes and may contribute to their damage. This will favor the development of new strategies to limit propagation of neurodegeneration.

Recent evidence indicates that genetically engineered EVs can be used as effective therapeutic vehicles for RNA interference and drug delivery in immune and tumor therapy (39-42). Therefore, in addition to providing a new strategy to limit disease propagation, improved understanding of EV trafficking outside cells by the use of optical manipulation will also greatly benefit the design of new drug delivery systems.

\section{Author contributions}

I.P., C.V., R.F, G.L., and D. C. designed the research. I.P. and L.A. performed experiments. D.C, R.F, L.A., and I.P. analyzed data. C.V. and D.C. wrote the paper. All authors revised and approved the final manuscript.

\section{Acknowledgments}

This work was financed in part by Fondazione Italiana Sclerosi Multipla (FISM

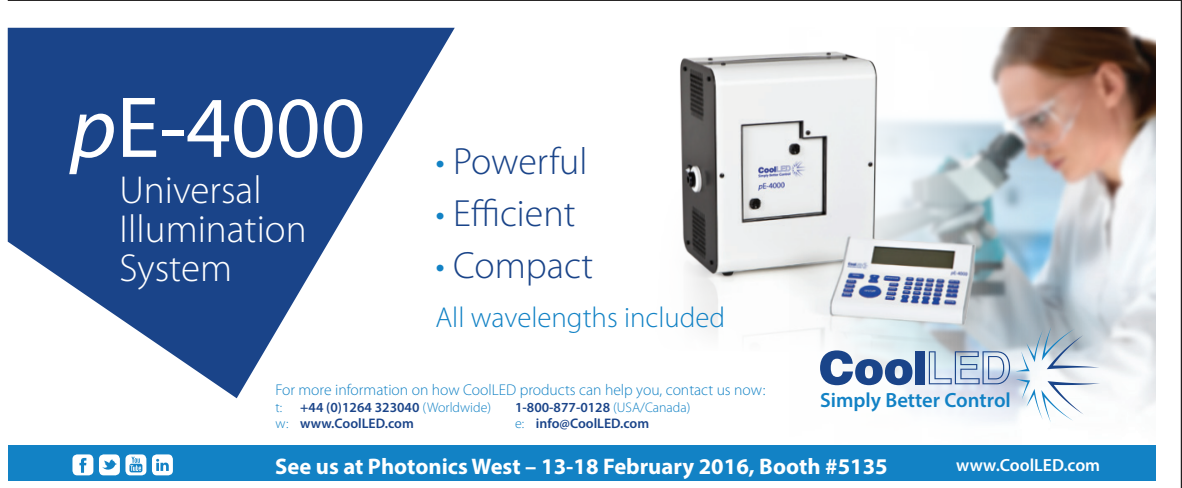

2012/R/17 to C.V.). I. P. was supported by a Fondazione Umberto Veronesi Fellowship (2015). We thank M. Gabrielli for assistance in some experiments.

\section{Competing interests}

The authors declare no competing interests.

\section{References}

1. Cocucci, E. and J. Meldolesi. 2015. Ectosomes and exosomes: shedding the confusion between extracellular vesicles. Trends Cell Biol. 25:364-372.

2. Lo Cicero, A., P.D. Stahl, and G. Raposo. 2015. Extracellular vesicles shuffling intercellular messages: for good or for bad. Curr. Opin. Cell Biol. 35:69-77.

3. Morelli, A.E., A.T. Larregina, W.J. Shufesky, M.L. Sullivan, D.B. Stolz, G.D. Papworth, A.F. Zahorchak, A.J. Logar, et al. 2004. Endocytosis, intracellular sorting, and processing of exosomes by dendritic cells. Blood 104:3257-3266.

4. Fitzner, D., M. Schnaars, D. van Rossum, G. Krishnamoorthy, P. Dibaj, M. Bakhti, T. Regen, U.K. Hanisch, and M. Simons. 2011. Selective transfer of exosomes from oligodendrocytes to microglia by macropinocytosis. J. Cell Sci. 124:447458.

5. Feng, D., W.L. Zhao, Y.Y. Ye, X.C. Bai, R.Q. Liu, L.F. Chang, Q. Zhou, and S.F. Sui. 2010. Cellular internalization of exosomes occurs through phagocytosis. Traffic 11:675-687.

6. Montecalvo, A., A.T. Larregina, W.J. Shufesky, and D.B. Stolz. 2012. Mechanism of transfer of functional microRNAs between mouse dendritic cells via exosomes. Blood 119:756-766.

7. Parolini, I., C. Federici, C. Raggi, L. Lugini, S. Palleschi, A. De Milito, C. Coscia, E. lessi, et al. 2009. Microenvironmental $\mathrm{pH}$ is a key factor for exosome traffic in tumor cells. J. Biol. Chem. 284:34211-34222

8. Mulcahy, L.A., R.C. Pink, and D.R. Carter. 2014. Routes and mechanisms of extracellular vesicle uptake. J Extracell Vesicles. 3.

9. Antonucci, F., E. Turola, L. Riganti, M. Caleo, M. Gabrielli, C. Perrotta, L. Novellino, E. Clementi, et al. 2012. Microvesicles released from microglia stimulate synaptic activity via enhanced sphingolipid metabolism. EMBO J. 31:1231-1240.

10. Gabrielli, M., N. Battista, L. Riganti, I. Prada, F. Antonucci, L. Cantone, M. Matteoli, M. Maccarrone, and C. Verderio. 2015. Active endocannabinoids are secreted on extracellular membrane vesicles. EMBO Rep. 16:213-220.

11. Vingtdeux, V., N. Sergeant, and L. Buée. 2012. Potential contribution of exosomes to the prion-like propagation of lesions in Alzheimer's disease. Front Physiol. 3:229.

12. Aguzzi, A. and L. Rajendran. 2009. The transcellular spread of cytosolic amyloids, prions, and prionoids. Neuron 64:783-790.

13. Schneider, A. and M. Simons. 2013. Exosomes: vesicular carriers for intercellular communication in neurodegenerative disorders. Cell Tissue Res. 352:33-47.

14. Minciacchi, V.R., M.R. Freeman, and D. Di Vizio. 2015. Extracellular Vesicles in Cancer: Exosomes, Microvesicles and the Emerging Role of Large Oncosomes. Semin. Cell Dev. Biol. 40:41-51.

15. Nakano, I., D. Garnier, M. Minata, and J. Rak. 2015. Extracellular vesicles in the biology of brain 
tumour stem cells - Implications for inter-cellular communication, therapy and biomarker development. Semin. Cell Dev. Biol. 40:17-26.

16. Candelario, K.M. and D.A. Steindler. 2014. The role of extracellular vesicles in the progression of neurodegenerative disease and cancer. Trends Mol. Med. 20:368-374.

17. Coleman, B.M. and A.F. Hill. 2015. Extracellular vesicles--Their role in the packaging and spread of misfolded proteins associated with neurodegenerative diseases. Semin. Cell Dev. Biol. 40:89-96.

18. Verderio, C., L. Muzio, E. Turola, A. Bergami, L. Novellino, F. Ruffini, L. Riganti, I. Corradini, et al. 2012. Myeloid microvesicles are a marker and therapeutic target for neuroinflammation. Ann. Neurol. 72:610-624.

19. Prada, I., R. Furlan, M. Matteoli, and C. Verderio. 2013. Classical and unconventional pathways of vesicular release in microglia. Glia 61:1003-1017.

20. Joshi, P., E. Turola, A. Ruiz, A. Bergami, D.D. Libera, L. Benussi, P. Giussani, G. Magnani, et al. 2014. Microglia convert aggregated amyloidbeta into neurotoxic forms through the shedding of microvesicles. Cell Death Differ. 21:582-593.

21. Agosta, F., D. Dalla Libera, E.G. Spinelli, A. Finardi, E. Canu, A. Bergami, L. Bocchio Chiavetto, M. Baronio, et al. 2014. Myeloid microvesicles in cerebrospinal fluid are associated with myelin damage and neuronal loss in mild cognitive impairment and Alzheimer disease. Ann. Neurol. 76:813-825

22. Kettenmann, H., U.K. Hanisch, M. Noda, and A. Verkhratsky. 2011. Physiology of microglia. Physiol. Rev. 91:461-553.

23. Turola, E., R. Furlan, F. Bianco, M. Matteoli, and C. Verderio. 2012. Microglial microvesicle secretion and intercellular signaling. Front Physiol. 3:149.

24. Volterra, A. and J. Meldolesi. 2005. Astrocytes, from brain glue to communication elements: the revolution continues. Nat. Rev. Neurosci. 6:626-640.

25. Ashkin, A., J.M. Dziedzic, J.E. Bjorkholm, and S. Chu. 1986. Observation of a single-beam gradient force optical trap for dielectric particles. Opt. Lett. 11:288.

26. Grier, D.G. 2003. A revolution in optical manipulation. Nature 424:810-816.

27. D’Este, E., G. Baj, P. Beuzer, E. Ferrari, G. Pinato, E. Tongiorgi, and D. Cojoc. 2011. Use of optical tweezers technology for long-term, focal stimulation of specific subcellular neuronal compartments. Integr Biol (Camb). 3:568-577.

28. Pinato, G., D. Cojoc, L.T. Lien, A. Ansuini, J. Ban, E. D'Este, and V. Torre. 2012. Less than 5 Netrin-1 molecules initiate attraction but 200 Sema3A molecules are necessary for repulsion. Sci Rep. 2:675.

29. Pinato, G., T. Raffaelli, E. D’Este, F. Tavano, and D. Cojoc. 2011. Optical delivery of liposome encapsulated chemical stimuli to neuronal cells. J. Biomed. Opt. 16:095001.

30. Bianco, F., C. Perrotta, L. Novellino, M. Francolini, L. Riganti, E. Menna, L. Saglietti, E.H. Schuchman, et al. 2009. Acid sphingomyelinase activity triggers microparticle release from glial cells. EMBO J. 28:1043-1054.

31. Al-Nedawi, K., B. Meehan, J. Micallef, V. Lhotak, L. May, A. Guha, and J. Rak. 2008. Intercellular transfer of the oncogenic receptor EGFRvIll by microvesicles derived from tumour cells. Nat. Cell Biol. 10:619-624.

32. Helmuth, J.A., C.J. Burckhardt, P. Koumoutsakos, U.F. Greber, and I.F. Sbalzarini. 2007. A novel supervised trajectory segmentation algorithm identifies distinct types of human adenovirus motion in host cells. J. Struct. Biol. 159:347-358.

33. Caspi, A., O. Yeger, I. Grosheva, and A.D. Bershadsky. 2001. A new dimension in retrograde flow: centripetal movement of engulfed particles. Biophys. J. 81:1990-2000.

34. de Bruin, K., N. Ruthardt, K. von Gersdorff, R. Bausinger, E. Wagner, M. Ogris, and C. Brauchle. 2007. Cellular dynamics of EGF receptor-targeted synthetic viruses. Mol. Ther. 15:1297-1305

35. Miyanishi, M., K. Tada, M. Koike, Y. Uchiyama, and T. Kitamura. 2007. Identification of Tim4 as a phosphatidylserine receptor. Nature 450:435-439.

36. Nolte-'t, E.N., S.I. Buschow, S.M. Anderton, W. Stoorvogel, and M.H. Wauben. 2009. Activated T cells recruit exosomes secreted by dendritic cells via LFA-1. Blood 113:1977-1981.

37. Tian, T., Y. Zhu, F. Hu, Y. Wang, N. Huang, and Z. Xiao. 2013. Dynamics of exosome internalization and trafficking. J. Cell. Physiol. 228:14871495.

38. Solon, J., P. Streicher, R. Richter, F. BrochardWyart, and P. Bassereau. 2006. Vesicles surfing on a lipid bilayer: self-induced haptotactic motion. Proc. Natl. Acad. Sci. USA 103:12382-12387.

39. El-Andaloussi, S., Y. Lee, S. Lakhal-Littleton, J. Li, Y. Seow, C. Gardiner, L. Alvarez-Erviti, I.L. Sargent, and M.J. Wood. 2012. Exosome- mediated delivery of siRNA in vitro and in vivo. Nat. Protoc. 7:2112-2126.

40. Zocco, D., P. Ferruzzi, F. Cappello, W.P. Kuo, and S. Fais. 2014. Extracellular vesicles as shuttles of tumor biomarkers and anti-tumor drugs. Front Oncol. 4:267.

41. Mizrak, A., M.F. Bolukbasi, G.B. Ozdener, G.J. Brenner, S. Madlener, E.P. Erkan, T. Strobel, X.O. Breakefield, and O. Saydam. 2013. Genetically engineered microvesicles carrying suicide mRNA/protein inhibit schwannoma tumor growth. Mol Ther. 21:101-108.

42. György, B., M.E. Hung, X.O. Breakefield, and J.N. Leonard. 2015. Therapeutic applications of extracellular vesicles: clinical promise and open questions. Annu. Rev. Pharmacol. Toxicol. 55:439-464.

Received 14 August 2015; accepted 29 September 2015.

Address correspondence to Claudia Verderio, CNR - Institute of Neuroscience, Via Vanvitelli 32, 20129 Milan, Italy; or Dan Cojoc, CNR - Institute of Materials, Optical Manipulation Lab, Area Science ParkBasovizza, S.S. 14, km 163.5, 34149 Trieste, Italy. E-mail: c.verderio@in.cnr.it or cojoc@iom.cnr.it.

To purchase reprints of this article, contact: biotechniques@fosterprinting.com

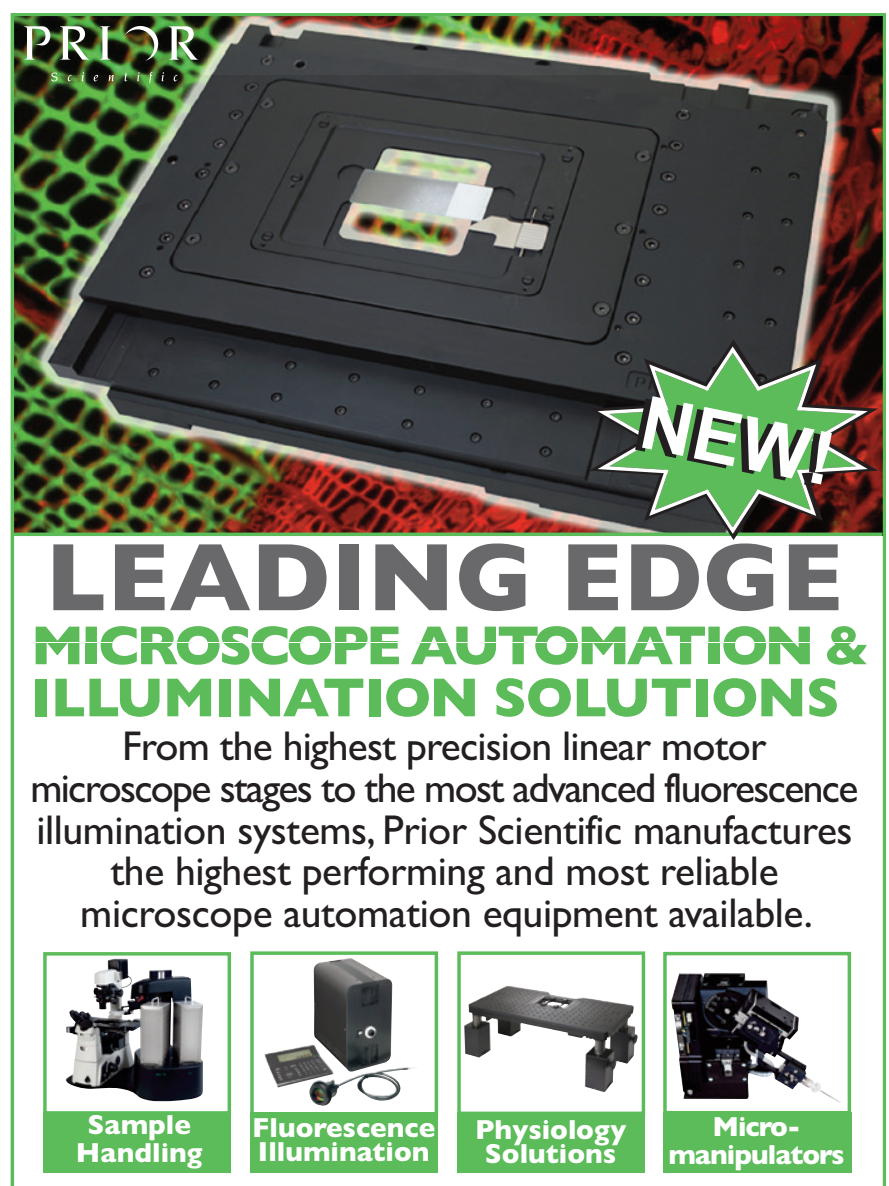

Prior Scientific, Inc. 80 Reservoir Park Dr. Rockland, MA. 02370 Tel: 800-877-2234 Web: www.prior.com 